\title{
RNase H-dependent amplification improves the accuracy of rolling circle amplification combined with loop-mediated isothermal amplification (RCA-LAMP)
}

\author{
Takema Hasegawa $^{\text {Corresp., }}{ }^{,}$, Diana Hapsari ${ }^{1}$, Hitoshi Iwahashi ${ }^{2}$ \\ ${ }^{1}$ The United Graduate School of Agricultural Science, Gifu University, Gifu, Gifu, Japan \\ 2 Faculty of Applied Biological Sciences, Gifu University, Gifu, Gifu, Japan \\ Corresponding Author: Takema Hasegawa \\ Email address: h5takema@gmail.com
}

The hybrid method upon combining rolling circle amplification and loop-mediated isothermal amplification (RCA-LAMP) was developed to quantify very small amount of different type of RNAs, such as miRNAs. RCA-LAMP can help detect short sequences through padlock probe (PLP) circularization and exhibit powerful DNA amplification. However, one of the factors that determines the detection limit of RCA-LAMP is nonspecific amplification. In this study, we improved the accuracy of RCA-LAMP through applying RNase H-dependent PCR (rhPCR) technology. In this method, the non-specific amplification was suppressed by using the rh primer, which is designed through blocking the modification at the $3^{\prime}$ end to stop DNA polymerase reaction and replacing the $6^{\text {th }}$ DNA molecule from the end with RNA using RNase $\mathrm{H} 2$ enzyme. Traditional RCA-LAMP amplified the non-specific amplicons from linear PLP without a targeting reaction, while RCA-LAMP with rh primer and RNase $\mathrm{H} 2$ suppressed the non-specific amplification. Conversely, we identified the risk posed upon conducting PLP cyclization reaction using Splint $R$ ligase in the RNA-targeting step that occurred even in the RNA-negative condition, which is another factor determining the detection limit of RCA-LAMP. Therefore, this study contributes in improving the accuracy of RNA quantification using RCA-LAMP. 
1

2 RNase H-dependent amplification improves the

G।

3 accuracy of rolling circle amplification combined with

4 loop-mediated isothermal amplification (RCA-LAMP)

5

6

7 Takema Hasegawa ${ }^{1}$, Diana Hapsari ${ }^{1}$, Hitoshi Iwahashi ${ }^{2}$

8

$9{ }^{1}$ The United Graduate School of Agricultural Science, Gifu University, Gifu, Japan

$10{ }^{2}$ Faculty of Applied Biological Sciences, Gifu University, Gifu, Japan

11

12

13

Corresponding Author:

Takema Hasegawa $^{1}$

14

15

1-1 Yanagido, Gifu, 501-1193, Japan

16

Email address: h5takema@gmail.com

17 
18

19

20

21

22

23

24

25

26

27

28

29

30

31

32

33

34

35

36

37

38

39

40

41

42

43

44

45

46

47

48

49

50

51

52

53

54

55

56

57

\section{Abstract}

The hybrid method upon combining rolling circle amplification and loop-mediated isothermal amplification (RCA-LAMP) was developed to quantify a very small amount of different types of RNAs, such as miRNAs. RCA-LAMP can help detect short sequences through padlock probe (PLP) circularization and exhibit powerful DNA amplification. However, one of the factors that determine the detection limit of RCA-LAMP is non-specific amplification. In this study, we improved the accuracy of RCA-LAMP by applying RNase H-dependent PCR (rhPCR) technology. In this method, the non-specific amplification was suppressed using the rh primer, which is designed by blocking the modification at the 3' end to stop DNA polymerase reaction and replacing the $6^{\text {th }}$ DNA molecule from the end with RNA using RNase $\mathrm{H} 2$ enzyme. Traditional RCA-LAMP amplified the non-specific amplicons from linear PLP without a targeting reaction, while RCA-LAMP with rh primer and RNase H2 suppressed the non-specific amplification. Conversely, we identified the risk posed upon conducting PLP cyclization reaction using Splint R ligase in the RNA-targeting step that occurred even in the RNA-negative condition, which is another factor determining the detection limit of RCA-LAMP. Therefore, this study contributes to improving the accuracy of RNA quantification using RCA-LAMP.

\section{Introduction}

Isothermal amplification methods have been studied and applied to quantify RNA (Yan et al., 2014). For example, reverse transcription loop-mediated isothermal amplification (RTLAMP) has been used for the detection of RNA viruses (Fukuta et al., 2003; Curtis, Rudolph \& Owen, 2008). LAMP amplifies DNA from the rolling circle amplification (RCA), which is used for miRNA and mRNA detection (Jonstrup, Koch \& Kjems, 2006; Cheng et al., 2009; Christian, 2001; Deng, 2017). This method's characteristic feature that the DNA template has a loop structure at both ends (Notomi, 2000). RCA amplifies long-chain single-strand DNA from a circular single-stranded DNA template (Lizardi, 1998). One of the advantages of isothermal amplification is the fast DNA amplification speed, which is because an optimum temperature is maintained throughout the reaction (Karami, 2011).

Recently, RCA-LAMP hybrid method has been extensively studied and applied (Ruff, 2016; Tian, 2019). Figure 1 shows the mechanism of RCA-LAMP. First, the padlock probe (PLP), a single-strand DNA probe designed with complementary sequences at both ends, hybridizes to the target RNA, and then, both ends of PLP are ligated using ligase to circularize. Second, the strand-displacement DNA polymerase synthesizes long-chain single-strand DNA using circular PLP as a template. This long-chain single-stranded DNA exhibits a loop structure at the 5' end by using a specifically designed primer to form a loop structure. Third, many LAMP DNA templates are synthesized from long-chain single-strand DNA. LAMP reaction occurs for each LAMP DNA template.

The advantages of RCA-LAMP are that it can detect very short RNA molecules, such as miRNAs, and exhibits high amplification power. Conversely, the major limitation of RCALAMP is the low accuracy of amplification, as there is a risk of synthesizing the LAMP DNA 
58

59

60

61

62

63

64

65

66

67

68

69

70

71

72

73

74

75

76

77

78

79

80

81

82

83

84

85

86

87

88

89

90

91

92

93

94

95

96

97

template through non-specific DNA synthesis using linear PLP and primers. In previous studies, DNA amplification was confirmed in RNA-negative samples, used to determine the detection limit of RNA (Tian, 2019). To improve the sensitivity of RNA quantification using this method, it is important to improve RCA-LAMP accuracy. Therefore, in this study, we aimed to improve the accuracy of RCA-LAMP.

In this study, we used Splint R ligase to circularize PLP and target RNA. Splint R binds a DNA nick on the DNA-RNA complementary strand (Lohman, 2014). Some reports suggest that Splint R is more accurate than the traditional ligase when applied in RCA (Deng, 2017; Jin, 2016; Takahashi, 2018). Moreover, we used RNA Solutions by Qualitative Analysis (AIST, Japan) as the model target RNA. This standard RNA is designed based on human mRNA and exhibits low homology with natural sequences (Tong, 2006).

For this, we focused on the technique of RNase H-dependent PCR (rhPCR) (Dobosy, 2011). In this method, rh primer, which is designed to block modification at the 3' end to stop DNA polymerase reaction and replace the $6^{\text {th }}$ DNA molecule from the end with RNA using RNase $\mathrm{H} 2$ enzyme is used. RNase $\mathrm{H} 2$ recognizes a perfect complementary double-strand and cleaves RNA precisely where the rh primer binds. DNA polymerase can then synthesize DNA by removing the blocking modification. Therefore, rhPCR helps prevent non-specific amplification. For this reason, to improve the accuracy of RCA-LAMP, we applied rhPCR (Figure 1).

In this study, we first discuss the reason why non-specific amplification occurs in a traditional RCA-LAMP assay. Furthermore, we show that through applying the rhPCR technique, the accuracy of RCA-LAMP is improved.

\section{Materials \& Methods}

\section{Materials}

Splint R ligase, Bst 2.0 polymerase, and dNTP mix were purchased from New England Biolabs (NEB, Massachusetts, USA). RNase H2, from Integrated DNA Technologies (Iowa, USA), EvaGreen fluorescent dye from CosmoBio (Tokyo, Japan), and betaine from Fijufilm Wako Chemical (Osaka, Japan) were also purchased.

\section{Design of DNA and RNA sequences}

RNA Solutions by Qualitative Analysis was purchased from AIST (Ibaraki, Japan) (hereafter known as "standard RNA") to be used as target RNA. This standard RNA is developed Certified reference material (CRM) (National Metrology Institute of Japan (NMIJ), Japan 2013). This standard RNA is available in five different types, including 500-A, 500-B, and 500-C that are $533 \mathrm{nt}$ each and 1000-A, and 1000-B that are $1033 \mathrm{nt}$ each. In this study, we used 1000-B. Table 1 shows PLP and primer sequences. PLP was synthesized and purified using Fasmac (Kanagawa, Japan). Primers were synthesized and purified by Integrated DNA Technologies (Iowa, USA).

\section{DNA amplification using RCA-LAMP}


For PLP-mediated targeting, $1 \mu \mathrm{L}$ of $10 \mathrm{x}$ reaction buffer, $2 \mu \mathrm{L}$ of $10 \mathrm{nM}$ PLP, and $5 \mu \mathrm{L}$ of RNA were mixed. The mixture was incubated at $90^{\circ} \mathrm{C}$ for $1 \mathrm{~min}, 70{ }^{\circ} \mathrm{C}$ for $1 \mathrm{~min}$, and cooled to room temperature for $45 \mathrm{~min}$. After that, $2 \mu \mathrm{L}$ of Splint R ligase $(6.75 \mathrm{U} / \mu \mathrm{L})$ was added and incubated at $37^{\circ} \mathrm{C}$ for $30 \mathrm{~min}$ for ligation, inactivated at $65^{\circ} \mathrm{C}$ for $20 \mathrm{~min}$ and cooled at $4{ }^{\circ} \mathrm{C}$. For RCA-LAMP amplification, 1x reaction buffer, $1 \mathrm{mM}$ each dNTP, $6 \mathrm{mM} \mathrm{MgSO}_{4}, 0.8$ $\mathrm{M}$ betaine, $0.8 \mu \mathrm{M}$ forward and reverse primer, $4 \mathrm{U}$ Bst 2.0 polymerase, and $1 \mu \mathrm{L}$ of PLP ligation products were mixed and incubated at $68{ }^{\circ} \mathrm{C}$ for $3 \mathrm{~h}$. The primers were replaced with rh primer, and $50 \mathrm{mU}$ RNase $\mathrm{H} 2$ enzyme was added to run the rhPCR reaction for RCA-LAMP. Then, the DNA amplicons were analyzed using $2 \%$ agarose gel electrophoresis and stained with ethidium bromide.

Quick-Load Purple $1 \mathrm{~kb}$ Plus DNA Ladder (0.1.10.0 kb) purchased from New England Biolabs (NEB; Massachusetts, USA) was used as the DNA ladder marker. Also, 1x EvaGreen was added to quantify DNA amplification in real-time. The fluorescence intensity of the RCALAMP reaction system was monitored in real-time using the Applied Biosystems Step One Plus real-time PCR system (Thermo Fisher Scientific) for $3 \mathrm{~h}$ at intervals of $1 \mathrm{~min}$.

\section{DNA sequencing}

DNA amplicons were purified using the Fastgene gel/PCR extraction kit purchased from Nippon Genetics (Tokyo, Japan). The purified amplicons were provided to the Division of Genomics Research of Gifu University, which runs the DNA Sequencer ABI 3130 to read the sequences.

\section{Results}

\section{Amplification of non-specific amplicon in RCA-LAMP}

In this study, we used the standard RNA, designed as a poly (A) tail forming an mRNA, as the target RNA. Splint R ligase was used for the circularization of PLP. RCA-LAMP system was designed using only a few primers. Two types of primer sets were designed to elucidate the causes of non-specific amplification (Figure $2 \mathrm{AB}$ ). In primer set A, the forward primer targeted the middle portion of PLP, and the reverse primer targeted the sequence of RNA-targeting region. In primer set $\mathrm{B}$, the forward primer targeted the RNA-target region sequence and the reverse primer targeted the middle portion of PLP.

To elucidate the cause of non-specific amplification, we read a sequence of non-specific amplicons. DNA amplification using RCA-LAMP should occur from circular PLP rather than from linear PLP. However, DNA amplification was observed from the linear PLP for both the primer sets (Figure 2C). To develop a more sensitive RNA detection method using RCA-LAMP, it is crucial to suppress non-specific amplification. Figures $2 \mathrm{C}$ and 2D show the resulting sequence of the nonspecific amplicon. The sequence of non-specific amplicons generated using primer set A was random (Figure 2D), which means that the polymerase reaction started at a random location. It is speculated that in this reaction the LAMP reaction template, which has two stem-loops at both ends, was synthesized. Conversely, the sequence of non-specific amplicons 
138

139

140

141

142

143

144

145

146

147

148

149

150

151

152

153

154

155

156

157

158

159

160

161

162

163

164

165

166

167

168

169

170

171

172

173

174

175

176

177

generated using the primer set B exhibited clear peaks (Figure 2E). Also, it was replaced with the primer sequence at an unexpected position, that is from the middle of the PLP sequence, which means that DNA polymerase synthesized DNA from the 3' end of the primer using PLP as a template. The $4^{\text {th }}$ to $7^{\text {th }}$ base sequences on the $3^{\text {' }}$ end of the forward primer had complementary sequences at the site where non-specific amplification occurred on the PLP (Figure 2F). However, the 3' end of the forward primer was not complementary. Thus, it was speculated that the annealing of the four bases present in the middle of the primer onto PLP increased the risk of non-specific amplification. These results indicate that it is difficult to improve the specificity of the reaction only by changing the sequence of the primers.

\section{rhPCR technique improves the specificity of RCA-LAMP}

To suppress non-specific amplification, we utilized rhPCR technique. For this, RNase H2 enzyme and rh primer with blocking modification at the 3' end and $6^{\text {th }}$ DNA from the 3' end replaced with RNA are used. RNase H2 cleaves the replaced RNA only when its periphery forms a complete complementary strand. The blocking modification is released from the primer when the RNA is cleaved, following the amplification reaction stars. The DNA amplification reaction initiates only when the primer is accurately annealed to the target region. Therefore, rhPCR was expected to suppress the initiation of non-specific amplification in RCA-LAMP and stop the subsequent amplification reaction even if non-specific amplification occurs.

Since the junction region sequence of PLP gets excised when Splint R ligase is used, the specificity can be improved by designing the rh primer's target region targeting the junction region of PLP. The rh primer should work as a primer for DNA template generated from circular PLP. If non-specific amplification occurs, the rh primer should stop the amplification reaction. The reverse primer of primer set A was designed using the rh primer because the target region was designed to target the junction region of PLP (Figure 3A). The forward primer of primer set $A$ is not targeted to the junction region of PLP. However, the rh primer has the potential to stop the non-specific amplification (Figure 3B).

Therefore, the reaction systems in which the reverse primer was replaced with rh primer and both the primers were replaced with rh primer were applied to conduct RCA-LAMP.

Simultaneously, the forward primer of primer set B was designed to target the junction region of PLP. Replacing this primer with the rh primer will make the 3' end of the rh primer complementary to the four bases of the 3' end of PLP. This complementary sequence of both 3' ends could lead to DNA polymerase reaction initiation from the 3' end of PLP. The newly synthesized double-stranded DNA provides a target for RNase H2, and blocking of the modification of rh primer is released. Therefore, primer set B was not replaced with rh primer.

Figure 3C shows the DNA amplification from each PLP using RCA-LAMP with rh primer. Traditional RCA-LAMP was used to amplify DNA from linear PLP. RCA-LAMP with rh primer did not amplify DNA from linear PLP (reaction time was $3 \mathrm{~h}$ ), and showed that rh primer suppressed the non-specific amplification of the linear PLP. Therefore, this result suggests that the rh primer contributed in improving the amplification accuracy of RCA-LAMP. 
178

179

180

181

182

183

184

185

186

187

188

189

190

191

192

193

194

195

196

197

198

199

200

201

202

203

204

205

206

207

208

209

210

211

212

213

214

215

216

217

\section{Real-time quantification using RCA-LAMP with rh primer}

The analytical performance of RCA-LAMP with rh primer was evaluated by detecting standard RNA at different concentrations. The samples with Splint R ligase reaction without RNA and Splint R ligase and RNA (linear PLP) were also evaluated. Figure 4A-C shows that the real-time fluorescence curves upon DNA amplification changed with standard RNA concentrations in the range of $1 \mathrm{nM}$ to $100 \mathrm{fM}$. DNA amplification was in proportion to the concentration of target RNA. Non-specific amplification from linear PLP (Splint R (-), RNA (-)) was suppressed using the rh primer. DNA was amplified from PLP, which reacted with Splint R ligase without RNA sample (RNA (-)). This result suggests that Splint R ligated the ends of DNA without RNA.

Figure 4D shows the relationship between $\mathrm{Ct}$ value and target RNA concentration. The amplification speed of the traditional RCA-LAMP using rh (-, -) was the fastest, and using rh $(+,+)$ was the slowest. The coefficient of determination $\left(\mathrm{R}^{2}\right)$ of the calibration curve within $1 \mathrm{nM}$ to $1 \mathrm{pM}$ of RCA-LAMP using rh $(+,-)$, rh $(+,+)$, and rh $(-,-)$ were $0.999,0.977$, and 0.732 , respectively. RCA-LAMP using $\mathrm{rh}(+,-)$ was the most accurate. The limit of detection of RCALAMP using rh (+, -) was 1 pM. Non-specific amplicons from linear PLP (Splint R (-), RNA (-)) with traditional RCA-LAMP using rh(-,-) were amplified at various speeds (Figure 4D). The rh primer could accurately suppress the non-specific amplification.

\section{Discussion}

In this study, we used the technique of rhPCR to improve the accuracy of RCA-LAMP. We showed that rh primer and RNase H2 enzyme suppressed the non-specific amplification of linear PLP.

RCA-LAMP using rh primer undergoes two kinds of enzyme reactions. First, the cleavage of the RNA portion complementary to the rh primer through RNase H2. Second, DNA amplification through strand-displacement DNA polymerase (here we used Bst DNA polymerase). It is considered that the speed of DNA amplification using $\mathrm{rh}(+,+)$ primer set is slower than that using $\mathrm{rh}(+,-)$ as the amplification mediated by $\mathrm{rh}(+,+)$ requires more RNase $\mathrm{H} 2$ enzyme than $\mathrm{rh}(+,-)$. Therefore, the reaction may be speedier if the amount of RNase H2 is increased; however it would make the cost high, and also, the accuracy needs to be examined.

The calibration curve of amplification using rh $(+,-)$ was more accurate than that using $\mathrm{rh}(+,+)$. The reason is that the amplification using $\mathrm{rh}(+,-)$ exhibits a more direct reaction system than that using rh $(+,+)$. Since the forward primer used is a regular primer, RCA reaction is quick. Amplification using rh $(+,-)$ could only generate an accurate calibration curve up to $1 \mathrm{pM}$. For this, the following two reasons are projected. One is that the reaction of RCA-LAMP is too robust, and thus, it poses the risk of amplifying non-specific sequences. The other is that the reaction of RCA-LAMP with rh primer is complex. For instance, it is not easy to estimate the amount of RNA and the DNA amplification rate in a reaction with many steps. These could be the attributing reasons that the amplification using $\mathrm{rh}(+,-)$ exhibits a more accurate calibration

Peer) reviewing PDF | (2021:03:58987:1:0:NEW 4 Jun 2021) 
218 curve than that using $\mathrm{rh}(+,+)$. Therefore, it is considered that the traditional RCA-LAMP could

219

220

221

222

223

224

225

226

227

228

229

230

231

232

233

234

235

236

237

238

239

240

241

242

243

244

245

246

247

248

249

250

251

252

253

254

255

256

not produce an accurate calibration curve due to non-specific amplification.

Splint R ligase was used to circularize PLP. However, DNA was amplified from PLP in a reaction with Splint R to RNA. Conversely, DNA was not amplified from PLP in a reaction without Splint R and RNA. This result indicates that Splint R poses a risk of executing the reaction without the presence of RNA. It has previously been reported that Splint R is more sensitive than other DNA ligases as it can detect RNA directly (Deng, 2017; Jin, 2016;

Takahashi, 2018). Therefore, it is important to study how Splint $\mathrm{R}$ reaction does not react negatively with RNA to improve the accuracy.

A calibration curve could not be generated using rhPCR for small amounts of RNA; however, the difference between circular and linear PLP could be assessed using RCA-LAMP. Thus, it can be applied for digital quantification of RNA because digital quantification can disregard the DNA amplification speed and consider only positive or negative amplification (Vogelstein \& Kinzler, 1999; Hindson, 2011; Quan, Sauzade \& Brouzes, 2018). RCA-LAMP combined with rh primer results in robust DNA amplification and can determine PLP cyclization associated with digital quantification. For this, we need to improve the accuracy of targeting RNA using PLP.

\section{Conclusions}

The technique of rhPCR suppressed the non-specific amplification from linear PLP in RCA-LAMP. It contributes to the improving signal-to-noise ration of RNA quantification by RCA-LAMP.

\section{Acknowledgements}

We would like to thank Editage (www.editage.jp) for English language editing.

\section{References}

Cheng Y, Zhang X, Li Z, Jiao X, Wang Y, Zhang Y. 2009. Highly sensitive determination of microRNA using target-primed and branched rolling-circle amplification. Angewandte Chemie (International ed. in English) 48(18):3268-3272.

Christian AT, Pattee MS, Attix CM, Reed BE, Sorensen KJ, Tucker JD. Detection of DNA point mutations and mRNA expression levels by rolling circle amplification in individual cells.

Proceedings of the National Academy of Sciences of the United States of America 98(25):1423814243.

Curtis KA, Rudolph DL, Owen SM. 2008. Rapid detection of HIV-1 by reverse-transcription, loop-mediated isothermal amplification (RT-LAMP). Journal of virological methods 151(2):264-270. 
257 Deng R, Zhang K, Sun Y, Ren X, Li J. 2017. Highly specific imaging of mRNA in single cells 258 by target RNA-initiated rolling circle amplification. Chemical science 8(5):3668-3675.

259 Dobosy JR, Rose SD, Beltz KR, Rupp SM, Powers KM, Behlke MA, Walder JA. 2001. RNase 260 H-dependent PCR (rhPCR): improved specificity and single nucleotide polymorphism detection 261 using blocked cleavable primers. BMC biotechnology 11:80.

262 Fukuta S, Iida T, Mizukami Y, Ishida A, Ueda J, Kanbe M, Ishimoto Y. 2003. Detection of 263 Japanese yam mosaic virus by RT-LAMP. Archives of virology 148(9):1713-1720.

264 Hindson BJ, Ness KD, Masquelier DA, Belgrader P, Heredia NJ, Makarewicz AJ, Bright IJ, 265 Lucero MY, Hiddessen AL, Legler TC, Kitano TK, Hodel MR, Petersen JF, Wyatt PW, 266 Steenblock ER, Shah PH, Bousse LJ, Troup CB, Mellen JC, Wittmann DK, Erndt NG, Cauley 267 TH, Koehler RT, So AP, Dube S, Rose KA, Montesclaros L, Wang S, Stumbo DP, Hodges SP, 268 Romine S, Milanovich FP, White HE, Regan JF, Karlin-Neumann GA, Hindson CM, Saxonov S, 269 Colston BW. 2011. High-throughput droplet digital PCR system for absolute quantitation of 270 DNA copy number. Analytical chemistry 83(22):8604-8610

271 Jin J, Vaud S, Zhelkovsky AM, Posfai J, McReynolds LA. 2016. Sensitive and specific miRNA 272 detection method using SplintR Ligase. Nucleic acids research 44(13):e116.

273 Jonstrup SP, Koch J, Kjems J. 2006. A microRNA detection system based on padlock probes and 274 rolling circle amplification. $R N A$ 12(9):1747-1752.

275 Karami A, Gill P, Motamedi MHK, Saghafinia M. 2011. A review of the current isothermal 276 amplification techniques: applications, advantages and disadvantages. Journal of Global 277 Infectious Diseases 3(3) 293-302.

278 Lizardi PM, Huang X, Zhu Z, Bray-Ward P, Thomas DC, Ward DC. 1998. Mutation detection 279 and single-molecule counting using isothermal rolling-circle amplification. Nature genetics 280 19(3):225-32

281 Lohman GJ, Zhang Y, Zhelkovsky AM, Cantor EJ, Evans TC Jr. 2014. Efficient DNA ligation in 282 DNA-RNA hybrid helices by Chlorella virus DNA ligase. Nucleic acids research 42(3):18312831844.

284 Notomi T, Okayama H, Masubuchi H, Yonekawa T, Watanabe K, Amino N, Hase T. 2000. 285 Loop-mediated isothermal amplification of DNA. Nucleic acids research 28(12):E63.

286 Quan PL, Sauzade M, Brouzes E. 2018. dPCR: A Technology Review. Sensors (Basel, 287 Switzerland) 18(4):1271.

288 Ruff LE, Fecteau JF, Uzri D, Messmer BT. 2006. Combining Isothermal Amplification 289 Techniques: Coupled RCA-LAMP. In: Demidov V, ed. Rolling Circle Amplification (RCA). 290 Switzerland: Springer, 57-63.

291 Takahashi H, Ohkawachi M, Horio K, Kobori T, Aki T, Matsumura Y, Nakashimada Y, 292 Okamura Y. 2018. RNase H-assisted RNA-primed rolling circle amplification for targeted RNA 293 sequence detection. Scientific reports 8(1):7770.

294 Tian W, Li P, He W, Liu C, Li Z. 2019. Rolling circle extension-actuated loop-mediated isothermal 295 amplification (RCA-LAMP) for ultrasensitive detection of microRNAs. Biosensors \& 296 bioelectronics 128:17-22. 
297 Tong W, Lucas AB, Shippy R, Fan X, Fang H, Hong H, Orr MS, Chu TM, Guo X, Collins PJ, 298 Sun YA, Wang SJ, Bao W, Wolfinger RD, Shchegrova S, Guo L, Warrington JA, Shi L. 2006. 299 Evaluation of external RNA controls for the assessment of microarray performance. Nature 300 biotechnology 24(9):1132-1139.

301 Vogelstein B, Kinzler KW. 1999. Digital PCR. Proceedings of the National Academy of Sciences 302 of the United States of America 96: 9236-9241.

303 Yan L, Zhou J, Zheng Y, Gamson AS, Roembke BT, Nakayama S, Sintim HO. 2014. Isothermal 304 amplified detection of DNA and RNA. Molecular bioSystems 10(5):970-1003. 
Figure 1

Schematic illustration of the RCA-LAMP applied rhPCR. The triangles indicate the PLP junction.

\section{Target RNA}

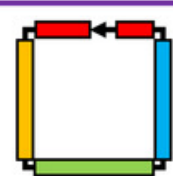

PLP

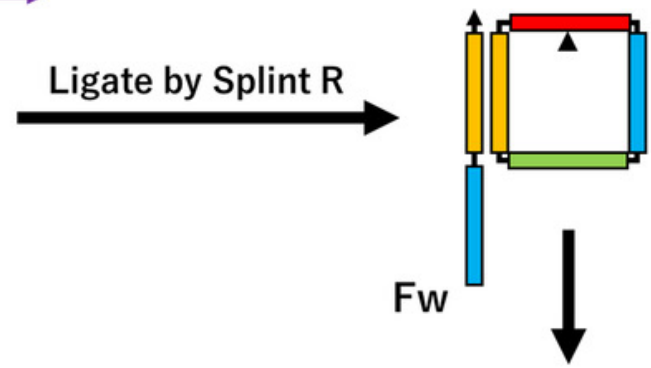

RCA
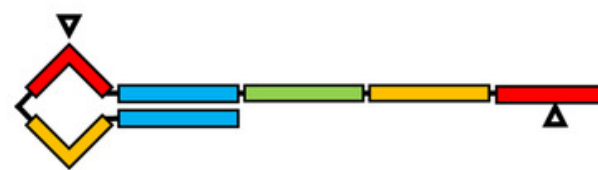

$\Delta$
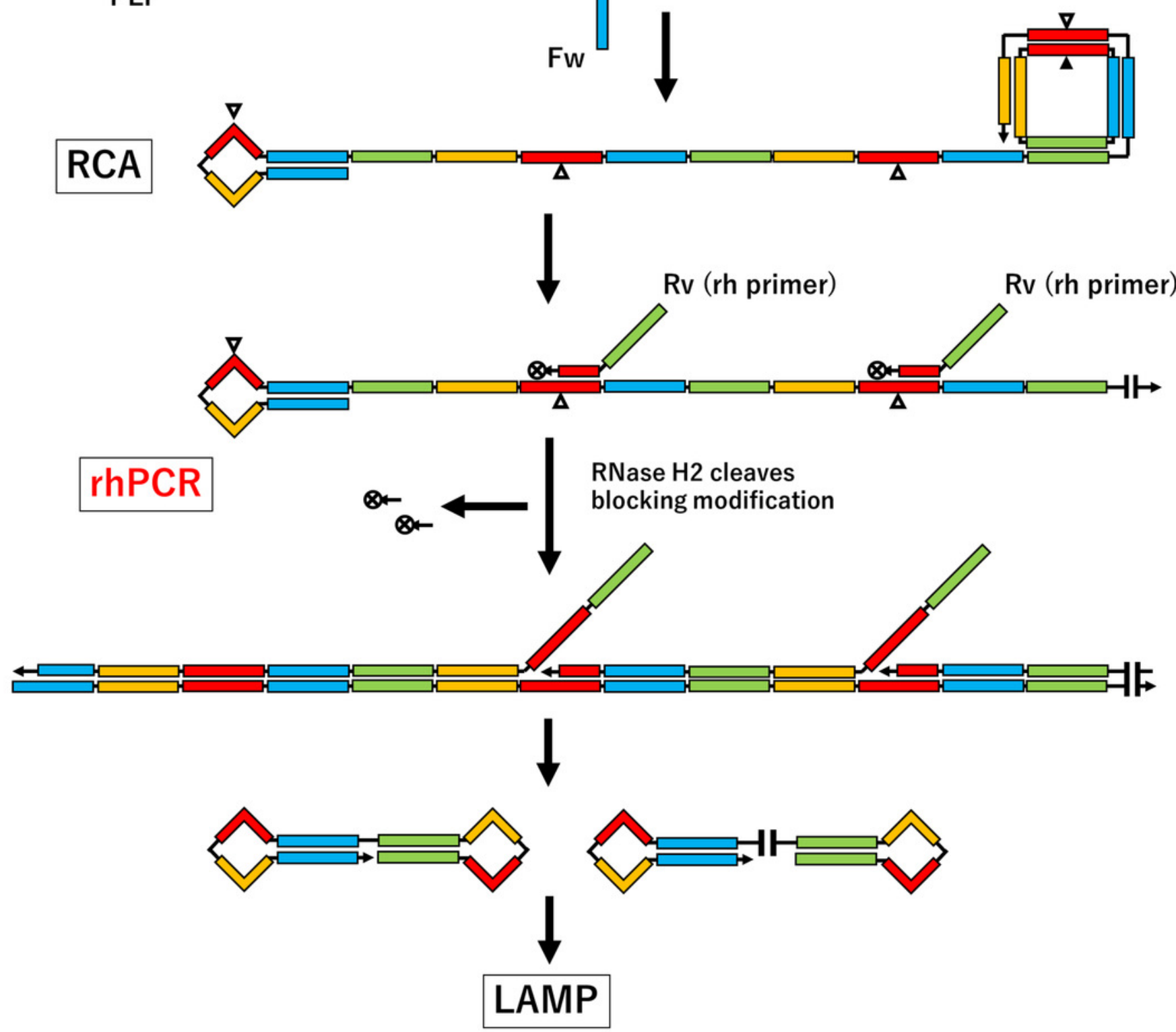


\section{Figure 2}

DNA amplification using traditional RCA-LAMP and the sequence of non-specific amplicon.

(A) Design of the primer set A. (B) Design of the primer set B. Small letter c: complimentary sequence; Fw: Forward primer; Rv: Reverse primer; T5' and T3': RNA targeting region. (C) DNA amplicon generated using RCA-LAMP with primer sets A and B. M: DNA ladder marker. (D) Sequence of amplicon generated using linear PLP with primer set A. (E) Sequence of amplicon generated using linear PLP with primer set B. (F) The sequence of PLP and forward primer of the primer set $B$. 


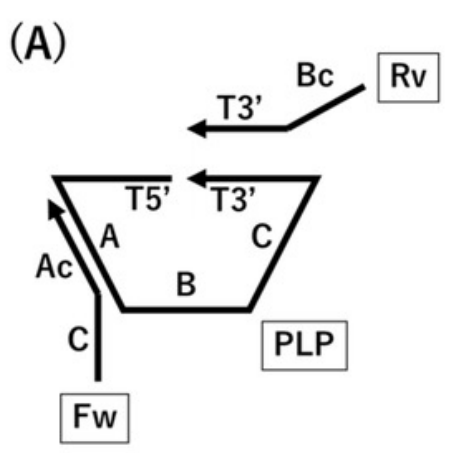

(C)

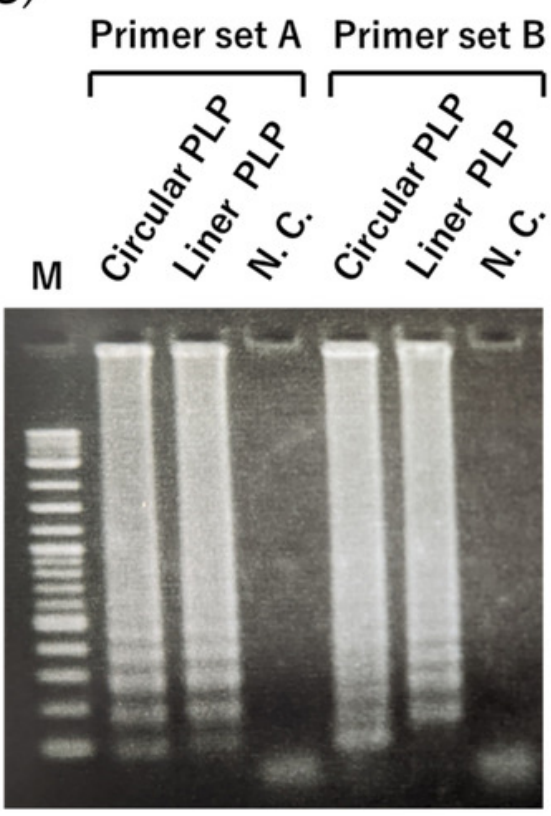

(B)

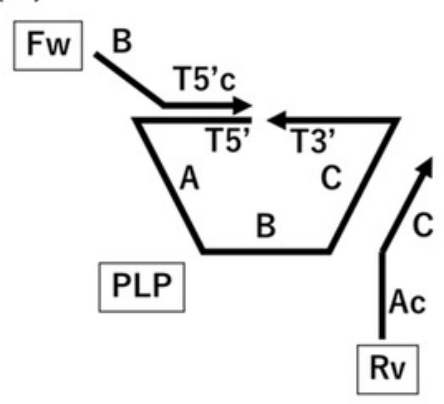

(D)

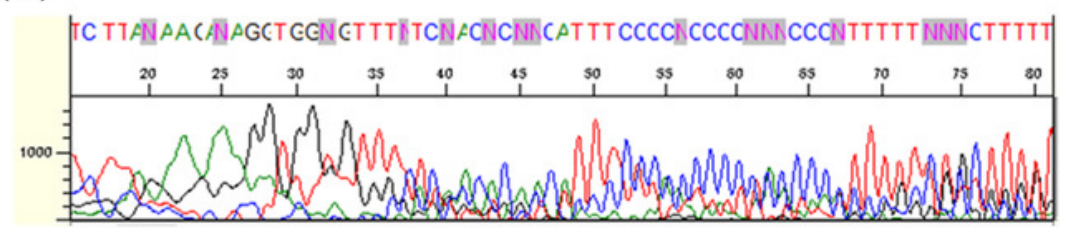

(E)

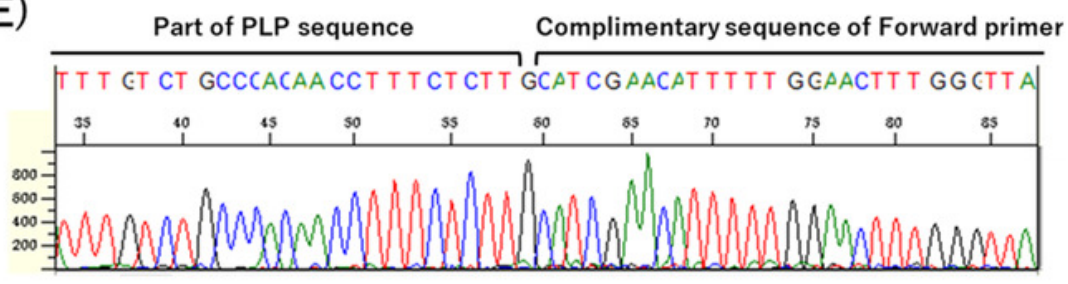

(F)

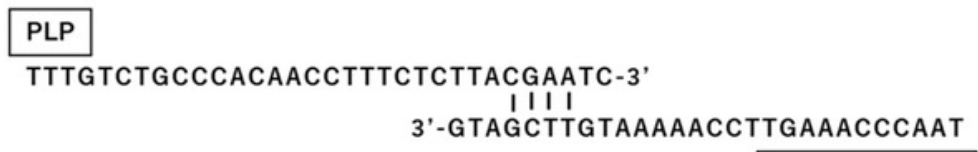


Figure 3

Figure 3. rhPCR technique improves specificity of RCA-LAMP.

(A) Primer designed with rh primer to replace reverse primer was called rh $(+,-)$. (B) Primer designed with rh primer to replace both primers was called $\mathrm{rh}(+,+)$. (C) Amplicon generated using RCA-LAMP with rh primer. M indicates the DNA ladder marker. $C$ indicates the sample in which circular PLP was used as a template DNA. L indicates the sample in which linear PLP was used as a template DNA. 
(A)

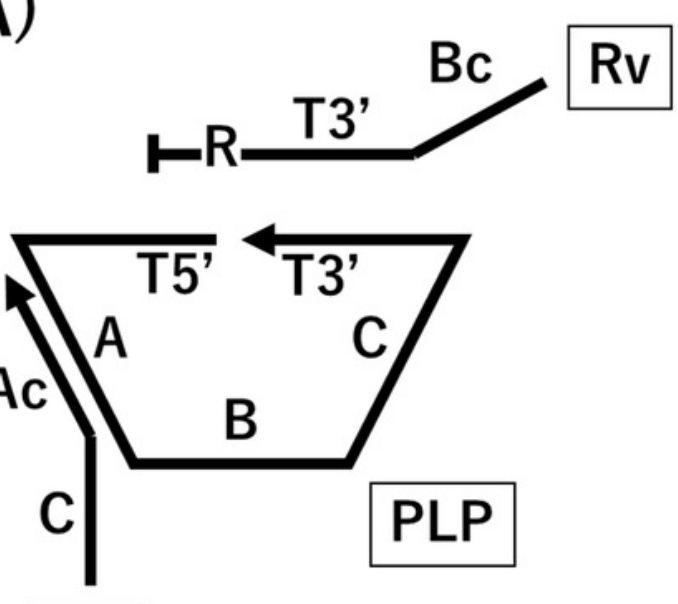

Fw
(B)<smiles></smiles>

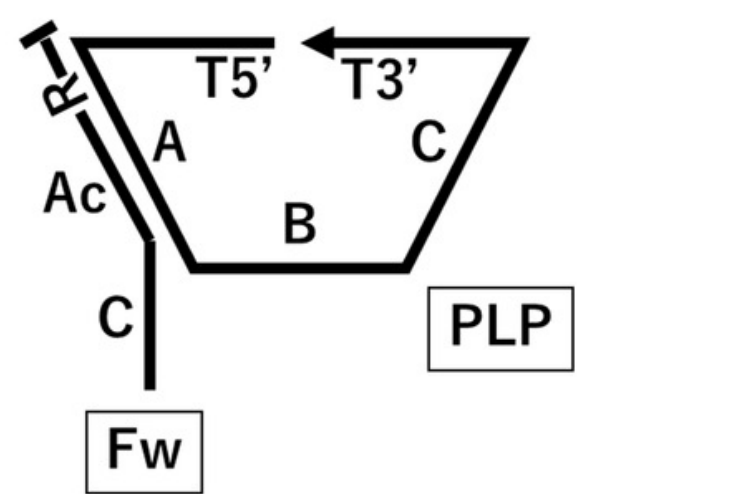

(C)
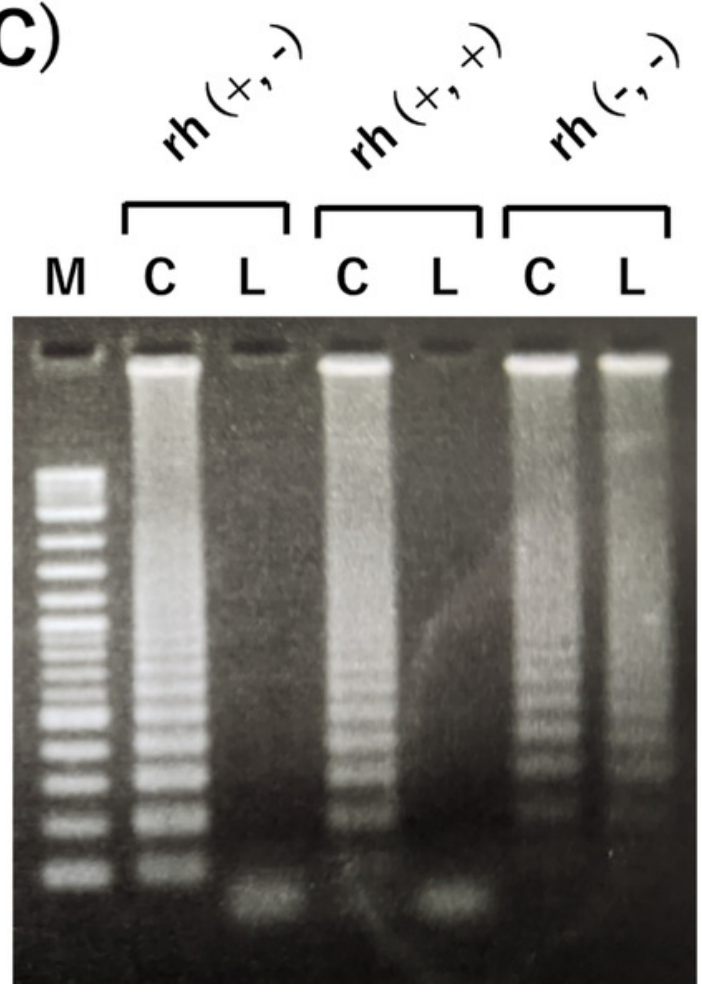
Figure 4

Real-time quantification using RCA-LAMP with rh primer.

(A) RCA-LAMP with rh(+, -). (B) RCA-LAMP with rh $(+,+)$. (C) RCA-LAMP with rh(-,-). (D) Ct value of each primer set. $r h(+,+)$ and $r h(+,-)$ had no value in the RNA(-) ligase (-) sample because there was no amplification $(n=3)$.

(A)

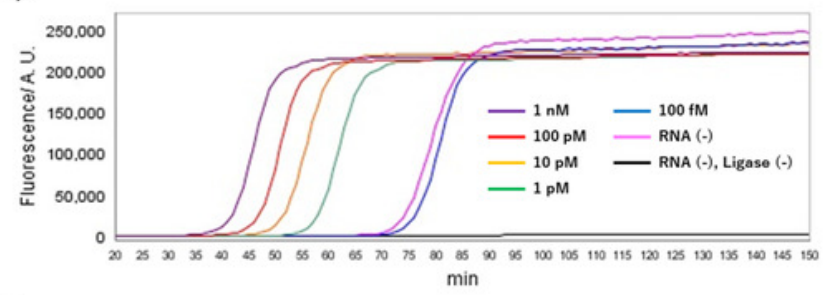

(B)

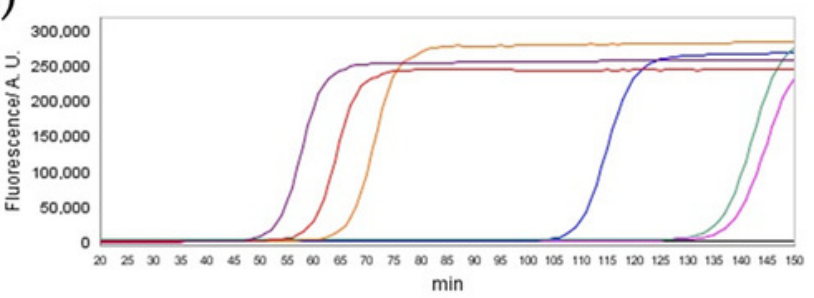

(C)

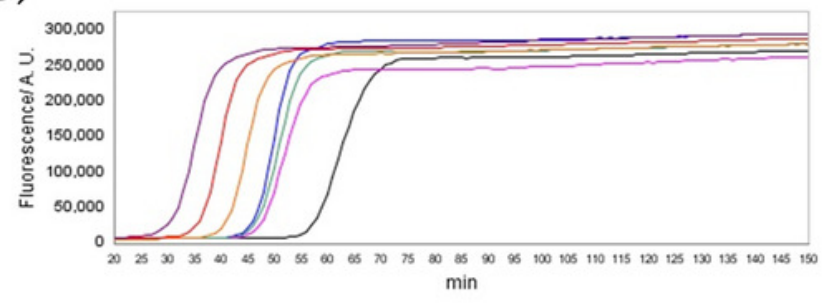

(D)

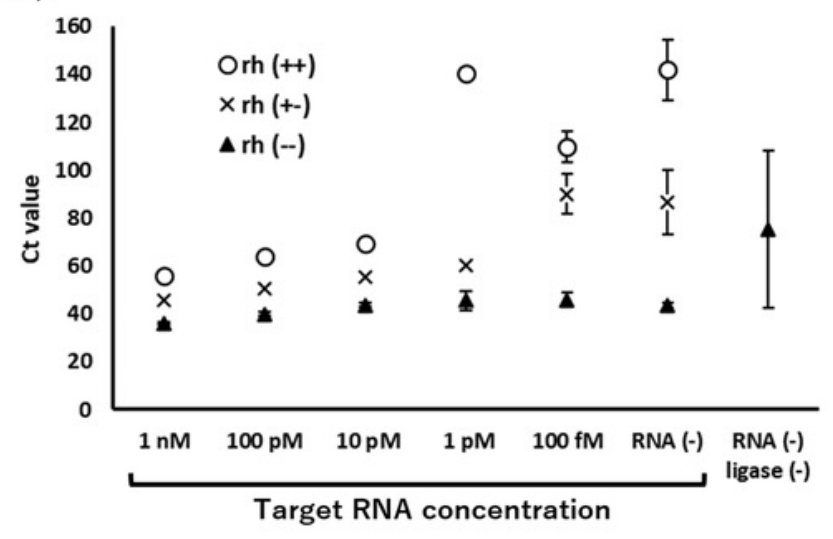




\section{Table $\mathbf{1}$ (on next page)}

Table 1. Padlock probe and primers sequence.

Underlines are homologous to the ribonucleotide sequence. $\mathrm{rA}$ and $\mathrm{rG}$ are RNA. $\mathrm{X}$ is $\mathrm{C} 3$ spacer. 
1

\begin{tabular}{|l|l|}
\hline & Sequence \\
\hline Padlock probe & $\begin{array}{l}\text { phos-GCATCGAACATTTTTGGAACTCTGCTCGACAAACGAC } \\
\text { ACGACACGACATTTCCCTAACCCTAACCCATTTGTCTGCCC } \\
\text { ACAACCTTTCTCTTACGAATC }\end{array}$ \\
\hline Primer set A Fw & CCCTAACCCATTTGTCTGCTTTGTTTGTCGAGCAGAGTTCC \\
\hline Primer set A Rv & GGGAAATGTCGTGTCGTGAAACACAACCTTTCTCTTACGAATC \\
\hline Primer set B Fw & CACGACATTTCCCTAACCCAAAGTTCCAAAAATGTTCGATG \\
\hline Primer set B Rv & GTGTCGTTTGTCGAGCAGTTTATTTGTCTGCCCACAACC \\
\hline rh primer Fw & $\begin{array}{l}\text { CCCTAACCCATTTGTCTGCTTTGTTTGTCGAGCAGAGTTCCrAAAAA } \\
\text { C-X }\end{array}$ \\
\hline rh primer Rv & $\begin{array}{l}\text { GGGAAATGTCGTGTCGTGAAACACAACCTTTCTCTTACGAATCrGC } \\
\text { ATCC-X }\end{array}$ \\
\hline
\end{tabular}

2 